\title{
Scheduling electric vehicles with shared charging stations
}

\author{
A. H. N. Galapitage ${ }^{1} \quad$ P. Pudney ${ }^{2}$
}

(Received 8 January 2016; revised 31 August 2016)

\begin{abstract}
Zimbabwe has one of the highest maternal death rates in the world, partly because of the difficulty in getting expectant mothers from rural villages to health care facilities. Specifically designed electric vehicles, called African Solar Taxis, are currently being developed for a rural hospital in Zimbabwe. The vehicles will be charged at solar charging stations located at the hospital and at heath clinics. The vehicles have limited speed and range, and charging is slow compared to conventional vehicles; because of this, efficient scheduling is important. In this article we describe a method for scheduling multiple taxis that share a common charging station.
\end{abstract}

DOI:10.21914/anziamj.v57i0.10443 gives this article, (c) Austral. Mathematical Soc. 2016. Published October 5, 2016, as part of the Proceedings of the 12th Biennial Engineering Mathematics and Applications Conference. ISSN 1445-8810. (Print two pages per sheet of paper.) Copies of this article must not be made otherwise available on the internet; instead link directly to the DOI for this article. Record comments on this article via http://journal . austms.org. au/ojs/index.php/ANZIAMJ/comment/add/10443/0 


\section{Contents}

1 Introduction

C209

2 Scheduling multiple taxis with no overlapping charging times

C211

3 Problem formulation

C213

4 Implementation

C217

5 Conclusion

C219

\section{Introduction}

The University of South Australia and a group of community volunteers are developing electric vehicles for transporting pregnant women to hospital in rural Zimbabwe [1]. The rugged, low-energy vehicles, called African Solar Taxis, will be recharged at charging stations that get their energy from photovoltaic panels, since grid-based electricity is either not available or unreliable.

The initial stage of the Solar Taxi project will be based at the St Albert's Mission Hospital, near the northern border of Zimbabwe. During the first stage of the project, taxis will collect women from four health clinics to the south of the hospital (Figure 1).

This article extends of the work of Albrecht and Pudney [2] on single taxi scheduling to schedules for multiple taxis that minimise the time required to collect women from health clinics and take them to the hospital. We consider two or more taxis where there is one charging station at the hospital that can charge at most one vehicle at a time. The taxis will be based at the hospital. Given a list of women waiting to be transported to the hospital, we design a 
Figure 1: Route of African solar taxis.

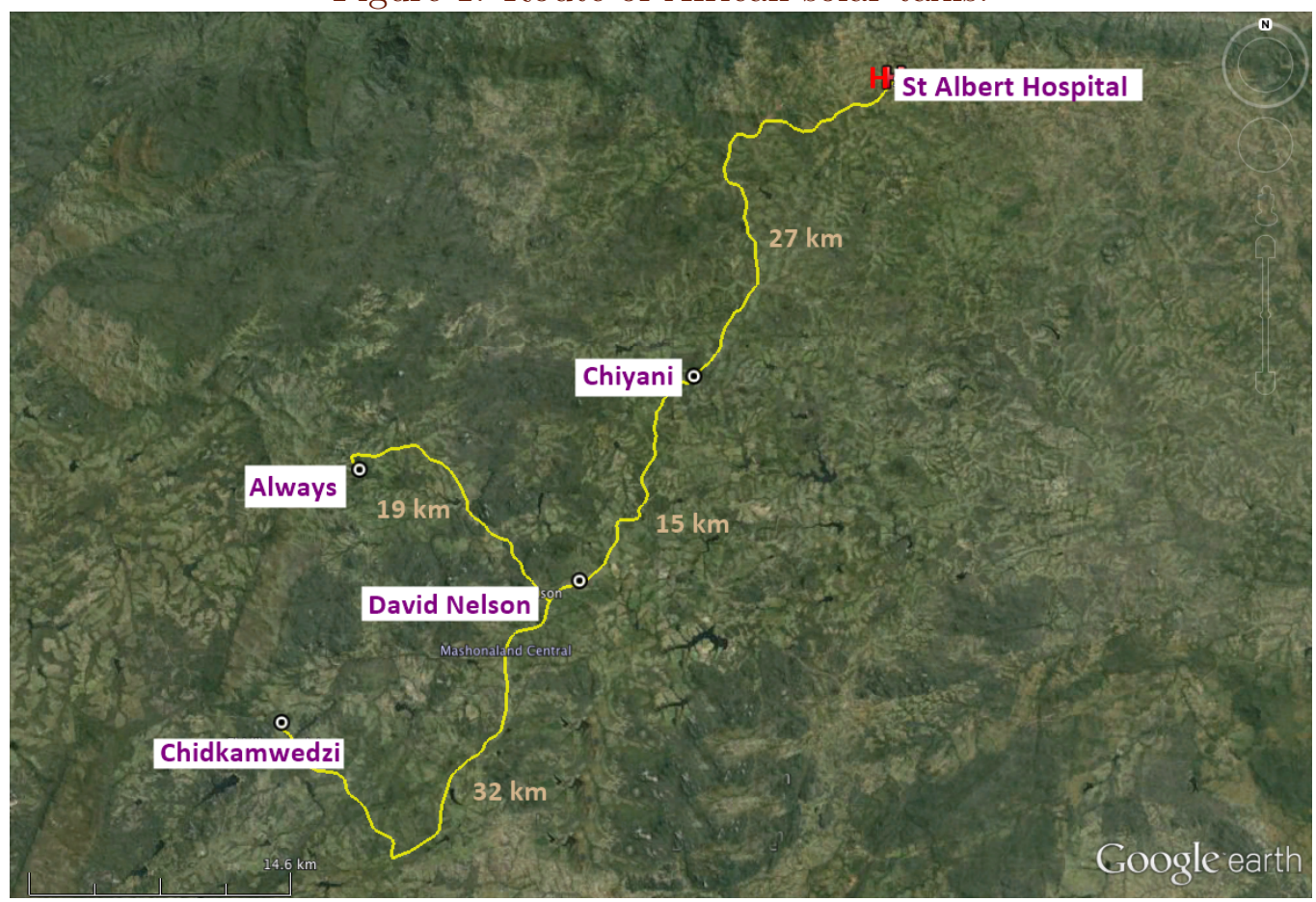

schedule for each taxi that minimises the time taken to transport the women while taking into account the constraints on vehicle charging.

Moghaddam [4] and Wang and Cheu [5] formulated and solved pickup and delivery problems for electric vehicles, but neither considered the case where the vehicles share a single charging station. 


\section{Scheduling multiple taxis with no overlapping charging times}

We wish to schedule multiple taxis to collect women from villages or health clinics, and bring them to the hospital. The trips that are required each day are specified. We need to determine which taxi does each trip, and the order of the trips, so that taxis can recharge at the hospital between trips. The aim is to minimise the time taken to complete a given set of trips. We need to take into account that at most one taxi is charging at any instant.

Trips start and finish at the hospital. Each trip has a known duration and a known energy requirement. Before embarking on a trip, each taxi has a charging session to ensure that it has enough energy in its battery for the trip.

We formulate the problem using a directed graph with nodes representing both charging sessions and trips. A vehicle alternates between charging nodes and driving nodes. We have one charging node for each driving node, so that vehicles have an opportunity for charging before each trip. However, charging sessions may have zero duration. In order to schedule taxis without charging overlaps we specify the order of charging nodes, and then place constraints on the ordered charging nodes to ensure that charging does not start on any charging node until charging has finished on the previous charging node. The driving nodes that follow each charging session are given by the minimum time solution.

Figure 2 illustrates a schedule with four trips and two vehicles. The vehicle that uses the first charging session does trip two and then finishes. The other vehicle does charging session two, trip one, charging session three, trip three, charging session four, trip four, and then finishes.

We also represent the problem using a $2 n+1$ by $2 n+1$ adjacency matrix where $n$ is the number of trips to be completed. The first row of the matrix represents the starting state, the next $\mathrm{n}$ rows represent charging sessions, 
Figure 2: Example diagram illustrating the model

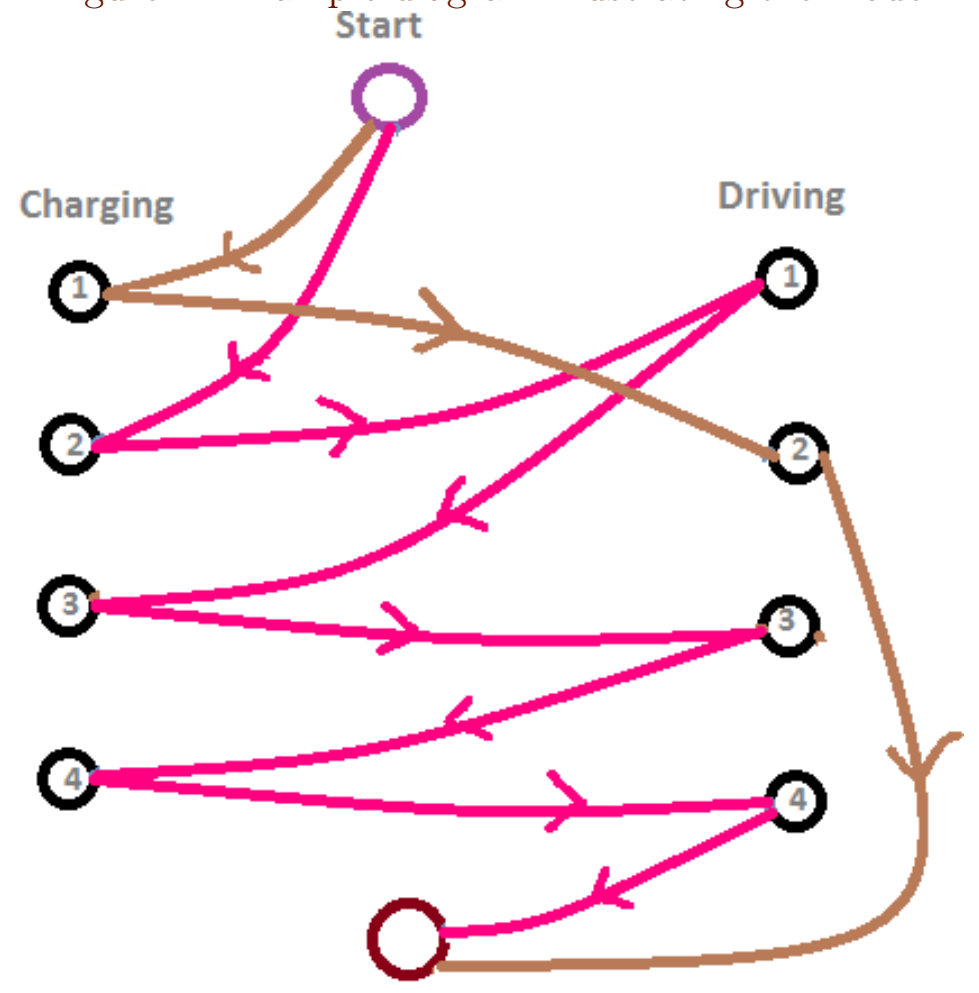

Finish

and the last $n$ rows represent driving. Moreover, the first $n$ columns are for charging nodes, the next $n$ columns are for driving nodes, and last column is for the finishing state. Figure 3 shows the matrix corresponding to the diagram in Figure 2. Section 3 uses this matrix representation to formulate the Mixed Integer Program model. 
Figure 3: Example matrix illustration the model. Charging Driving

Finish

\begin{tabular}{|c|c|c|c|c|c|c|c|c|c|}
\hline \multirow[b]{2}{*}{ Start } & \\
\hline & 1 & 1 & 0 & 0 & 0 & 0 & 0 & 0 & 0 \\
\hline \multirow{5}{*}{ 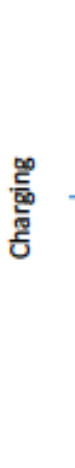 } & 0 & 0 & 0 & 0 & 0 & 1 & 0 & 0 & 0 \\
\hline & 0 & 0 & 0 & 0 & 1 & 0 & 0 & 0 & 0 \\
\hline & 0 & 0 & 0 & 0 & 0 & 0 & 1 & 0 & 0 \\
\hline & 0 & 0 & 0 & 0 & 0 & 0 & 0 & 1 & 0 \\
\hline & 0 & 0 & 1 & 0 & 0 & 0 & 0 & 0 & 0 \\
\hline \multirow{3}{*}{$\frac{\stackrel{D}{c}}{\sum^{2}}$} & 0 & 0 & 0 & 0 & 0 & 0 & 0 & 0 & 1 \\
\hline & 0 & 0 & 0 & 1 & 0 & 0 & 0 & 0 & 0 \\
\hline & 0 & 0 & 0 & 0 & 0 & 0 & 0 & 0 & 1 \\
\hline
\end{tabular}

\section{Problem formulation}

Let $n$ be the number of trips to be completed. We model the state of the taxis by states $j \in\{1, \ldots, 2 n+1\}$ where:

- $j=0$ represents the state of the system at the beginning of the day;

- $\mathfrak{j} \in\{1, \ldots, \mathfrak{n}\}$ represents a taxi doing charge session $\mathfrak{j}$;

- $j \in\{n+1, \ldots, 2 n\}$ represents a taxi doing trip $j-n$; 
- $s=2 n+1$ represents the state of the system at the end of the day.

The problem parameters are:

$D_{i}$ the driving duration of trip $i$;

B the energy capacity of the battery;

$p_{c}$ the energy charging rate of the battery;

$p_{d}$ the energy discharge rate of the battery while driving;

$\mathrm{V}$ the number of available vehicles.

The key decision variables are:

$x_{i j} \quad$ one if state $j$ immediately follows state $i$, zero otherwise;

$\mathrm{T}_{\text {csi }}$ the time that a taxi starts charging for trip $i$;

$\mathrm{T}_{\text {cfi }}$ the time that a taxi finishes charging for trip $i$;

$\mathrm{T}_{\mathrm{dsi}}$ the time that a taxi starts driving for trip $i$;

$\mathrm{T}_{\text {dfi }}$ the time that a taxi finishes driving for trip $i$;

$\mathrm{E}_{\mathrm{csi}}$ the energy in the battery at time $\mathrm{T}_{\mathrm{csi}}$;

$E_{c f i}$ the energy in the battery at time $T_{\text {cfi }}$;

$E_{d s i}$ the energy in the battery at time $T_{d s i}$;

$E_{d f i}$ the energy in the battery at time $T_{\text {dfi }}$;

$\mathrm{C}_{i}$ the charging duration at the beginning of trip $i$;

$\mathrm{S}$ the span (final arrival time back at the hospital) of the solution.

The objective is to minimise the span $S$ subject to the requirement that the span is not less than any trip finish time:

$$
S \geqslant T_{\text {dfi }} \quad \text { for all } i \in\{1, \ldots, n\} \text {. }
$$

Link constraints ensure that each taxi starts at the hospital, does each trip exactly once, and finishes back at the hospital. The start state must link to at most $V$ charge states:

$$
\sum_{j=1}^{n} x_{0 j} \leqslant v, \quad \sum_{j=1}^{n} x_{0 j} \geqslant 1, \quad \sum_{j=n+1}^{2 n+1} x_{0 j}=0 .
$$

The last of the above constraints ensures that each taxi starts with a charging session (which may have zero duration). There must be at most $V$ links from 
drive states to the finish state:

$$
\sum_{i=n+1}^{2 n} x_{i s} \leqslant v, \quad \sum_{i=n+1}^{2 n} x_{i s} \geqslant 1, \quad \sum_{i=1}^{n} x_{i s}=0 .
$$

Each charge state $i \in\{1, \ldots n$,$\} must link to exactly one drive state j \in$ $\{n+1, \ldots, 2 n\}$ :

$$
\sum_{j=n+1}^{2 n} x_{i j}=1 \quad \text { for all } i \in\{1, \ldots, n\} .
$$

Each drive state must link to either a charge state or the finish state:

$$
\sum_{j=1}^{n} x_{i j}+x_{i s}=1 \quad \text { for all } i \in\{n+1, \ldots, 2 n\} .
$$

The times at which each trip starts charging, finishes charging, starts driving, and finishes driving give the time constraints. The taxis start with a full battery and so the start state has

$$
\begin{aligned}
T_{\text {cfi }}=0 & \text { for all } i \in\{1, \ldots, n\} \text { and } x_{0 i}=1, \\
T_{\text {csi }}=0 & \text { for all } i \in\{1, \ldots, n\} \text { and } x_{0 i}=1, \\
T_{d s i}=0 & \text { for all } i \in\{1, \ldots, n\} \text { and } x_{0 i}=1
\end{aligned}
$$

Since the battery level is full before the first trip, there is no charging duration for the first trip and $C_{0}=0$. Charging duration depends on the difference between the required start energy of the new trip and the energy left after the previous trip:

$$
\begin{aligned}
C_{i} p_{c} & \geqslant E_{c f j}-E_{c s i} \quad \text { for all } i \in\{1, \ldots, n\}, \\
C_{i} & \geqslant 0 \text { for all } i \in\{2, \ldots, n\} .
\end{aligned}
$$


Charge should commence after finishing a trip or after waiting for an available charging session:

$$
\begin{aligned}
& T_{c s i} \geqslant T_{c s(i-1)}+C_{i-1} \quad \text { for all } i \in\{2, \ldots, n\}, \\
& T_{c s j} \geqslant T_{d f(i-n)} \text { for all } i \in\{n+1, \ldots, 2 n\}, j \in\{1, \ldots, n\} \text { and } x_{i j}=1 .
\end{aligned}
$$

For each trip, driving commences when charging is complete and driving finishes after the trip duration:

$$
\begin{aligned}
& T_{d s(j-n)}=T_{c s i}+C_{i} \quad \text { for all } i \in\{1, \ldots, n\}, j \in\{n+1, \ldots, 2 n\} \text { and } x_{i j}=1, \\
& T_{d f(i-n)}=T_{d s(i-n)}+D_{i-n} \quad \text { for all } i \in\{n+1, \ldots, 2 n\} .
\end{aligned}
$$

Driving cannot start until after charging has finished:

$$
\mathrm{T}_{\text {cfi }} \geqslant \mathrm{T}_{\mathrm{dsi}} \text { for all } i \in\{1, \ldots, \mathfrak{n}\} \text {. }
$$

The energy in the batteries control the energy constraints. The start energy of the first trip of each vehicle is the battery capacity:

$$
\begin{array}{ll}
E_{c f i}=0 & \text { for all } i \in\{1, \ldots, n\} \text { and } x_{0 i}=1, \\
E_{c s i}=0 & \text { for all } i \in\{1, \ldots, n\} \text { and } x_{0 i}=1, \\
E_{d s i}=0 & \text { for all } i \in\{1, \ldots, n\} \text { and } x_{0 i}=1
\end{array}
$$

Before a trip is started, there must be enough energy in the battery to complete the trip:

$$
\begin{array}{ll}
E_{d f i} \geqslant 0 & \text { for all } i \in\{1, \ldots, n\}, \\
E_{d s i} \leqslant B & \text { for all } i \in\{1, \ldots, n\}, \\
E_{d f i} \leqslant B & \text { for all } i \in\{1, \ldots, n\} .
\end{array}
$$

The remaining energy of the battery of each vehicle depends on the charge before starting the trip and the trip duration:

$$
\begin{aligned}
& E_{d f j}=E_{d s j}-D_{j} p_{d} \quad \text { for all } j \in\{1, \ldots, n\}, \\
& E_{c f j}=E_{c s j}+C_{j} p_{c} \text { for all } j \in\{1, \ldots, n\}, i \in\{1, \ldots, n\} \text { and } x_{i j}=1, \\
& E_{c s j}=E_{d f i} \quad \text { for all } i \in\{1, \ldots, n\}, j \in\{2, \ldots, n\} \text { and } x_{i j}=1, \\
& E_{d s j}=E_{c f i} \text { for all } i \in\{1, \ldots, n\}, j \in\{1, \ldots, n\} \text { and } x_{i j}=1
\end{aligned}
$$


Zero constraints such as equation (6) are also written using the "big- $M$ trick", which uses a large positive constant $M$ to ensure that the constraint is not tight [6]. For example, the first line of equation (6) is rewritten as

$$
M\left(x_{0 i}-1\right)+T_{\text {cfi }}=0 \quad \text { for all } i \in\{1, \ldots, n\} .
$$

Which, for a sufficiently large $M$ and when $x_{0 i}=1$, reduces to $T_{\text {cfi }}=0$.

\section{Implementation}

We implemented this model using MiniZinc [3]. The calculation is fast for small problems. For example, for a problem with four trips and two vehicles the solution is found in less than $30 \mathrm{~s}$.

Example Our example problem has two taxis to complete four trips, which have trip durations $D_{1}=7500 \mathrm{~s}, D_{2}=6500 \mathrm{~s}, D_{3}=5000 \mathrm{~s}$ and $D_{4}=7000 \mathrm{~s}$. The battery capacity of a vehicle is $20 \mathrm{MJ}$ which does an $80 \mathrm{~km}$ round trip in 2.6 hours. The battery charges at a rate $p_{c}=5000 \mathrm{~W}$, and so can be fully charged in just over an hour.

The schedule must avoid multiple taxis charging at one time and it has to satisfy all the conditions discussed in Section 3. Table 1 shows the solution for this example - one vehicle does trips one and four while the other vehicle does trips two and three. Table 1 shows the energy level of the battery for the optimal solution. The columns are:

- $\mathrm{T}_{\mathrm{cs}}$ the time that the taxi starts charging before a trip;

- $\mathrm{T}_{\mathrm{cf}}$ the time that the taxi finishes charging before a trip;

- $\mathrm{T}_{\mathrm{ds}}$ the time that the taxi starts driving;

- $\mathrm{T}_{\mathrm{df}}$ the time that the taxi finishes driving. 
Table 1: Time steps (in s) of the example schedule with two vehicles.

\begin{tabular}{rrrrr} 
Trip & $\mathrm{T}_{\mathrm{cs}}$ & $\mathrm{T}_{\mathrm{cf}}$ & $\mathrm{T}_{\mathrm{ds}}$ & $\mathrm{T}_{\mathrm{df}}$ \\
\hline 1 & 0 & 0 & 0 & 7500 \\
2 & 0 & 0 & 0 & 6500 \\
3 & 6500 & 8250 & 8250 & 11500 \\
4 & 8250 & 11500 & 11500 & 18500
\end{tabular}

Figure 4: Time line of the example schedule with two vehicles.

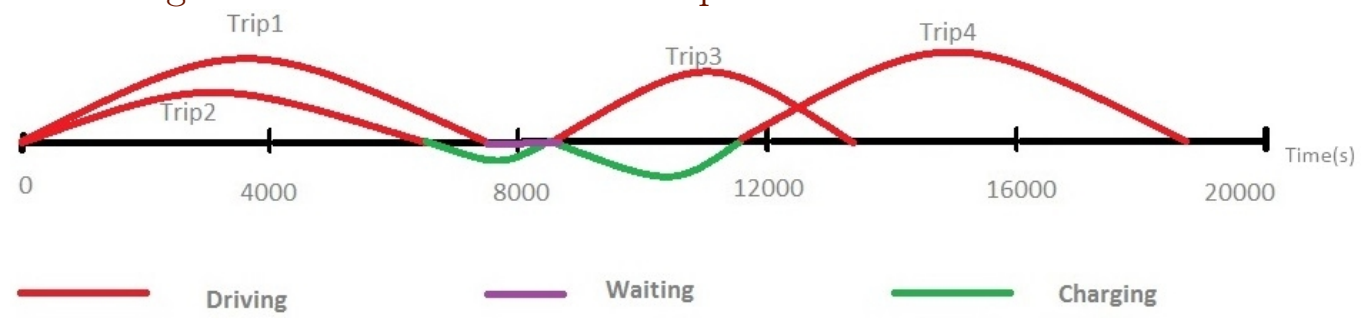

Figure 4 shows a time line of the schedule, where arcs above the axis represent driving and arcs below the axis represent charging. There is no overlapping of charging times; vehicle two finishes trip two and then starts charging while vehicle one returns to the hospital and waits for vehicle two to finish charging. This schedule has a total span of $18500 \mathrm{~s}$.

In each step of the schedule we keep track of the battery level of each vehicle as well as times of charging and driving. Table 2 shows the battery energy at various stages for the optimal solution. The columns are:

- $\mathrm{E}_{\mathrm{cs}}$ the energy that the taxi starts charging before a trip;

- $\mathrm{E}_{\mathrm{cf}}$ the energy that the taxi finishes charging before a trip;

- $E_{\mathrm{ds}}$ the energy that the taxi starts driving;

- $\mathrm{E}_{\mathrm{df}}$ the energy that the taxi finishes driving.

This optimal solution is not unique. For example, if vehicle one does trips 
Table 2: Battery energy in MJ for the example schedule with two vehicles.

\begin{tabular}{rrrrr} 
Trip & $E_{\text {cs }}$ & $E_{\text {cf }}$ & $E_{d s}$ & $E_{d f}$ \\
\hline 1 & 20 & 20 & 20 & 1.25 \\
2 & 20 & 20 & 20 & 3.75 \\
3 & 3.75 & 12.5 & 12.5 & 0 \\
4 & 1.25 & 17.5 & 17.5 & 0
\end{tabular}

one and three and vehicle two does trips two and four, then the span will be the same, but with trip three finishing last. It is possible to calculate all optimal solutions and allow a preferred optimal solution to be selected.

\section{Conclusion}

We formulated a problem of scheduling multiple electric vehicles with nonoverlapping charging sessions. For the African Solar Taxi problem, this model could be run at the start of each day to determine the best schedule for the day. It could also be used off-line to generate efficient schedules for different trip combinations.

Another application is scheduling electric kerb-side collection vehicles, such as garbage or recycling trucks, which return to the depot multiple times each day.

\section{References}

[1] African Solar Taxis. http://africansolartaxi.com. 2016 (cit. on p. C209). 
[2] Amie Albrecht and Peter Pudney. "Pick up and delivery with a solar-recharged vehicle". In: 22nd National Conference of the Australian Operations Research Society (ASOR 2013). http://www. asor. org. au/conferences/asor2013/J8/albrecht.pdf. Australian Society for Operations Research, 2013, pp. 239-246 (cit. on p. C209).

[3] Kim Marriott and Peter J. Stuckey. A MiniZinc Tutorial. Technical report. http://www.minizinc.org/downloads/doclatest/minizinc-tute.pdf. 2015 (cit. on p. C217).

[4] Navid Matin Moghaddam. "The partially rechargeable electric vehicle routing problem with time windows and capacitated charging stations". http://tigerprints.clemson.edu/cgi/viewcontent.cgi?article= 3210\&context=all_theses. PhD thesis. Clemson University, 2015 (cit. on p. C210).

[5] Hao Wang and Ruey Cheu. "Operations of a taxi fleet for advance reservations using electric vehicles and charging stations". In: Trans. Res. Rec.: J. Trans. Res. Board 2352 (2013). doi:10.3141/2352-01, pp. 1-10 (cit. on p. C210).

[6] H. Paul Williams. Model Building in Mathematical Programming. 4th. http://au.wiley.com/WileyCDA/WileyTitle/productCd1118443330.html. Wiley, 1999, pp. 154-187 (cit. on p. C217).

\section{Author addresses}

1. A. H. N. Galapitage, School of Mathematical Sciences, University of South Australia, South Australia 5095, Australia. mailto:galah0010unisa.edu.au

2. P. Pudney, School of Mathematical Sciences, University of South Australia, South Australia 5095, Australia. mailto:Peter. Pudney@unisa.edu. au 